\title{
Association Between Nonalcoholic Fatty Liver Disease and Incident Diabetes Mellitus among Japanese: A Retrospective Cohort Study Using Propensity-Score Matching
}

Xiaodan Zheng ( $\square$ zhengxiaodan512@163.com)

Peking University Shenzhen Hospital

Changchun Cao

Shenzhen Dapeng New District Nao'ao Hospital

Yongcheng He

Shenzhen Hengshen Hospital

Xinyu Wang

The First Affiliated Hospital of Shenzhen University

Jun Wu

Peking University Shenzhen Hospital

Haofei Hu

The First Affiliated Hospital of Shenzhen University

Research

Keywords: NAFLD, Incident diabetes, Propensity-score matching

Posted Date: February 26th, 2021

DOI: https://doi.org/10.21203/rs.3.rs-255620/v1

License: (9) (1) This work is licensed under a Creative Commons Attribution 4.0 International License. Read Full License 


\section{Abstract}

Background: Previous studies demonstrated nonalcoholic fatty liver disease (NAFLD) was a significant risk factor of diabetes mellitus (DM). However, these studies could not fully reflect the association between NAFLD and DM due to unbalanced confounding factors. We aimed to use propensity score matching (PSM) analysis to explore the actual association between NAFLD and DM in a large Japanese population cohort.

Methods: This was a retrospective PSM cohort study. A total of 14,271 Japanese participants without DM at baseline from Murakami Memorial Hospital were eventually enrolled in our study between 2004 and 2015. The baseline NAFLD and incidence of DM during follow-up were selected as the independent variable and outcome measure. To balance the confounding factors between 1,711 participants with NAFLD and 1,711 participants with non-NAFLD, we performed a nonparsimonious multivariable logistic regression for 1:1 PSM. Our study used the doubly robust estimation method to verify the correlation between NAFLD and DM.

Result: After PSM, the approximate incidence of DM in NAFLD participants and non-NAFLD participants were 907.082/100,000 person-years and 458.980/100,000 person-years, respectively. The risk of developing DM in participants with NAFLD increased by $92 \%$ in the PSM cohort (HR=1.92, 95\% confidence interval $(\mathrm{Cl}): 1.35-2.74, \mathrm{P}=0.0003)$. The participants with NAFLD were 2.15-fold more likely to develop diabetes than non-NAFLD participants in the PSM cohort after adjusting for the demographic and laboratory biochemical variables $(\mathrm{HR}=2.15,95 \% \mathrm{Cl}$ : $1.48-3.11, \mathrm{P}<0.0001)$. In the PSM cohort, the participants with NAFLD had an $89 \%$ increased risk of diabetes after adjustment of the propensity score $(\mathrm{HR}=1.89,95 \% \mathrm{Cl}: 1.33-2.69, \mathrm{P}=0.0004)$. In the subgroup analysis, most potential confounding variables did not influence the association between NAFLD and DM risk after PSM, except for body mass index ( $P$ for interaction=0.0140) and visceral fat obesity ( $P$ for interaction $=0.0157$ ). In the sensitivity analysis, participants with NAFLD had an $82 \%$ increase in the risk of DM in the original cohort $(\mathrm{HR}=1.82 \otimes 95 \% \mathrm{Cl}$ : 1.33-2.48, $\mathrm{P}=0.0001)$ and a $70 \%$ increase in the weighted cohort $(\mathrm{HR}=1.70 \otimes 95 \% \mathrm{Cl}: 1.40-2.06, \mathrm{P}<0.00001)$, respectively.

Conclusion: In the PSM cohort, the risk of developing DM in the participants with NAFLD increased by $92 \%$. In the sensitivity analysis, the risk of developing DM in the participants with NAFLD increased by $70 \%$ in the weighted cohort. Additionally, subgroup analysis showed that obese participants with NAFLD should be more concerned about the risk of diabetes.

\section{Introduction}

Diabetes mellitus (DM) became a serious global public health problem. According to global diabetes data, the prevalence of DM in 2019 was estimated at 9.3\% (approximately 500 million people)[1]. Diabetes mellitus and its complications seriously affected the health of patients and increased medical costs, which caused a heavy economic burden to patients and society[2]. DM is a metabolic disease characterized by hyperglycemia due to insufficient insulin secretion or insulin resistance (IR)[3]. The pathogenesis and risk factors of DM have been concerned and studied by many scholars.

Some prospective cohort studies recently reported that nonalcoholic fatty liver disease (NAFLD) was a significant risk factor for DM[4,5]. NAFLD is the most common cause of chronic liver disease, often accompanied by diabetes, obesity and hyperlipidemia[6, 7]. Liu et al.[8] revealed that the risk of NAFLD participants developing diabetes increased by $67 \%$ after adjustment for demographic and laboratory data. A prospective study involving 132,377 participants found that NAFLD was an independent risk factor for diabetes after adjusting for confounding variables[9]. In a recent meta-analysis of 33 studies involving more than 500,000 individuals and 27953 diabetic participants, participants with NAFLD increased the risk of developing diabetes by 1.19 times compared with those without NAFLD[10].

However, the traditional regression model in previous studies cannot avoid unmeasured or residual confounding and model overfitting, which might hinder the actual connection between NAFLD and DM. The participant's propensity score (PS) for NAFLD is the conditional probability of developing diabetes given a set of covariates measured at baseline. The propensity score method was widely applied in the studies of limited resources or inability to conduct randomized clinical trials, especially when outcomes of studies were rare in a large number of covariates[11]. Therefore, our study applied propensity score- 
matched analysis to explore the actual connection between NAFLD and DM in the NAGALA (NAfld in the Gifu Area, Longitudinal Analysis) database from 14,271 Japanese people.

\section{Methods}

\section{Study Design and Data Source}

The current study was a secondary analysis of the research on NAGALA in the publically available DRYAD databases (www.Datadryad.org database). The raw data was freely extracted from this site, provided by Okamura et al.[12] from Ectopic fat obesity presents the greatest risk for incident diabetes: a population-based longitudinal study. A total of 20,944 subjects who underwent medical examinations from 2004 to 2015 were contained in this database. All participants completed a detailed questionnaire to obtain their demographic data and health behaviors. Trained professionals measured related clinical data, such as body weight and waist circumference. Laboratory-related biochemical parameters were collected under the condition of standardization and processed following a unified process. As a retrospective cohort study, our study decreased the risk of selection bias and observation bias.

The authors of the original research gave up all copyrights of these data. Therefore, our study performed a secondary analysis on their database without prejudice to the authors' rights. Besides, the original research of Murakami Memorial Hospital was approved by the ethics committee, and all participants' informed consent was obtained.

\section{Study Sample}

In the original study, 5480 participants were excluded from 20,944 Japanese participants in the Murakami Memorial Hospital based on the following criteria: (1) viral hepatitis (defined by measurements of hepatitis B antigen and hepatitis $C$ antibody at baseline), (2) alcoholic fatty liver disease, (3) diagnosed as DM at baseline, (4) fasting plasma glucose $\geq 6.1 \mathrm{mmol} / \mathrm{L}$, (5) using any medication at baseline, (6) missing data of covariates. Finally, 15,464 participants were included in the original study. For

further analysis, 1184 participants with excessive alcohol consumption (alcohol consumption $>210 \mathrm{~g} /$ week in males and $>140$ $\mathrm{g} /$ week in females[13]) and 9 participants without HDL-C data were excluded in our study. Figure 1 detailed the selection process of all participants.

\section{Independent Variable And Covariates}

The independent variable in our study was baseline NAFLD. NAFLD was diagnosed by the findings of abdominal ultrasonography performed by trained technicians[12]. We are interested in the following covariates: age, gender, waist circumference (WC), body mass index (BMI), alcohol consumption, smoking status, regular exerciser, systolic blood pressure (SBP) at baseline, diastolic blood pressure (DBP) at baseline, aspartate aminotransferase(AST), alanine aminotransferase(ALT), total cholesterol(TC), gamma-glutamyl transferase (GGT), HbA1c, fasting plasma glucose (FPG), highdensity lipoprotein cholesterol(HDL-C), triglycerides(TG), and day of follow-up. Alcohol consumption was divided into three categories: no or very little alcohol consumption (less than 40 grams of alcohol per week), light alcohol consumption (40-140 grams of alcohol per week) and moderate alcohol consumption (140g-280 grams of alcohol per week)[14]. Participants were classified as regular exercisers when they regularly played any type of exercise at least once a week[15]. Visceral fat obesity was described as a $W C \geq 90 \mathrm{~cm}$ in males or $\geq 80 \mathrm{~cm}$ in females[16].

\section{Outcome Measure}

The outcome measure in our study was the presence of DM. DM was defined as HbA1c $\geq 6.5 \%, F P G \geq 7 \mathrm{mmol} / \mathrm{L}$ [17]or selfreport during follow-up. 


\section{Statistical Analyses}

Data conforming to the normal distribution were presented as mean \pm standard deviation (SD), while data conforming to the skewed distribution were described as median and quaternary ranges (25-75th percentile). Categorical variables were expressed as frequencies and percentages. The one-way ANOVA, the Kruskal-Wallis $\mathrm{H}$ test and the chi-square test were performed to detect differences between groups.

To adjust for the differences of the baseline characteristics between the NAFLD and non-NAFLD groups (Table 1) and to gather a group of participants with similar baseline characteristics, we used propensity score matching (PSM) for analysis. We performed a non-parsimonious multivariable logistic regression model to calculate the PS of 14271 participants, with NAFLD as the independent variable and 17 baseline characteristics as covariates. We used a 1:1 matching protocol without replacement (greedy matching algorithm) for matching, and the caliper width is equal to 0.01 . The evaluation index of the balance between groups was the standard deviation $[18,19]$. If the standard deviation was less than 0.1 , it was considered that the variables between the groups were well balanced[18, 19]. To describe the incidence, we calculated the person-days of the follow-up from baseline the date of the baseline interview to the date of incident DM or December 31, 2016, whichever came first[20]. 
Table 1

Baseline characteristics before and after propensity score matching.

\begin{tabular}{|c|c|c|c|c|c|c|}
\hline \multirow[t]{2}{*}{ Characteristic } & \multirow[t]{2}{*}{ non-NAFLD } & $\begin{array}{l}\text { Before } \\
\text { Matching }\end{array}$ & \multirow[t]{2}{*}{$\mathrm{SD}(100 \%)$} & \multirow[t]{2}{*}{ non-NAFLD } & \multirow{2}{*}{$\begin{array}{l}\text { After } \\
\text { Matching } \\
\text { NAFLD }\end{array}$} & \multirow[t]{2}{*}{$\operatorname{SD}(100 \%)$} \\
\hline & & NAFLD & & & & \\
\hline Participants & 11760 & \multicolumn{2}{|l|}{2511} & 1711 & \multicolumn{2}{|l|}{1711} \\
\hline Age(years) & $43.27 \pm 8.99$ & $44.79 \pm 8.32$ & 17.6 & $45.85 \pm 9.28$ & $45.36 \pm 8.28$ & 5.6 \\
\hline \multicolumn{2}{|l|}{ Gender } & & \multicolumn{2}{|l|}{78.2} & & 0.1 \\
\hline Male & $5398(45.90 \%)$ & \multicolumn{2}{|l|}{2033 (80.96\%) } & 1321 (77.21\%) & \multicolumn{2}{|l|}{1322 (77.26\%) } \\
\hline Female & $6362(54.10 \%)$ & \multicolumn{2}{|l|}{478 (19.04\%) } & 390 (22.79\%) & \multicolumn{2}{|l|}{$389(22.74 \%)$} \\
\hline BMI(Kg/m2) & $21.33 \pm 2.61$ & $25.50 \pm 3.13$ & 144.8 & $24.34 \pm 2.59$ & $24.41 \pm 2.43$ & 2.7 \\
\hline WC (cm) & $74.10 \pm 7.92$ & $85.98 \pm 7.78$ & 151.4 & $83.18 \pm 6.81$ & $83.35 \pm 6.36$ & 2.6 \\
\hline SBP (mmHg) & $111.93 \pm 14.02$ & $\begin{array}{l}123.44 \pm \\
14.84\end{array}$ & 79.7 & $120.93 \pm 14.06$ & $\begin{array}{l}120.88 \pm \\
14.34\end{array}$ & 0.4 \\
\hline DBP (mmHg) & $69.71 \pm 9.86$ & $77.83 \pm 10.19$ & 81.0 & $76.11 \pm 9.68$ & $76.09 \pm 9.73$ & 0.2 \\
\hline FPG (mg/dL) & $91.79 \pm 7.24$ & $97.19 \pm 6.55$ & 78.2 & $96.24 \pm 6.64$ & $96.30 \pm 6.63$ & 0.9 \\
\hline HbA1c (\%) & $5.15 \pm 0.31$ & $5.30 \pm 0.33$ & 46.3 & $5.27 \pm 0.33$ & $5.26 \pm 0.33$ & 1.5 \\
\hline ALT(U/L) & $\begin{array}{l}15.00 \\
(12.00,20.00)\end{array}$ & $\begin{array}{l}27.00 \\
(20.00,39.00)\end{array}$ & 95.7 & $21.00(16.00,28.50)$ & $\begin{array}{l}24.00 \\
(18.00,31.00)\end{array}$ & 3.7 \\
\hline AST(U/L) & $\begin{array}{l}17.00 \\
(14.00,20.00)\end{array}$ & $\begin{array}{l}20.00 \\
(17.00,26.00)\end{array}$ & 55.6 & $19.00(16.00,23.00)$ & $\begin{array}{l}19.00 \\
(16.00,23.00)\end{array}$ & 9.2 \\
\hline GGT(U/L) & $\begin{array}{l}14.00 \\
(11.00,18.00)\end{array}$ & $\begin{array}{l}23.00 \\
(16.00,33.00)\end{array}$ & 61.5 & $19.00(14.00,29.00)$ & $\begin{array}{l}20.00 \\
(15.00,28.00)\end{array}$ & 5.4 \\
\hline $\mathrm{TC}(\mathrm{mg} / \mathrm{dL})$ & $195.50 \pm 32.98$ & $\begin{array}{l}210.51 \pm \\
33.52\end{array}$ & 45.1 & $205.09 \pm 34.72$ & $\begin{array}{l}207.69 \pm \\
33.46\end{array}$ & 7.6 \\
\hline TG (mg/dL) & $58.00(40.00,84.00)$ & $\begin{array}{l}110.00 \\
(77.00,159.00)\end{array}$ & 95.9 & $93.00(63.00,135.00)$ & $\begin{array}{l}98.00 \\
(70.00,139.00)\end{array}$ & 1.9 \\
\hline $\begin{array}{l}\text { HDL- } \\
\mathrm{C}(\mathrm{mg} / \mathrm{dL})\end{array}$ & $58.71 \pm 15.34$ & $45.85 \pm 11.07$ & 96.2 & $47.65 \pm 12.16$ & $47.77 \pm 11.63$ & 0.9 \\
\hline $\begin{array}{l}\text { Smoking } \\
\text { status }\end{array}$ & & & 35.2 & & & 3.8 \\
\hline Never smoker & 7563 (64.31\%) & $1184(47.15 \%)$ & & 831 (48.57\%) & 803 (46.93\%) & \\
\hline Ever smoker & $1929(16.40 \%)$ & $640(25.49 \%)$ & & $422(24.66 \%)$ & 447 (26.13\%) & \\
\hline $\begin{array}{l}\text { Current } \\
\text { smoker }\end{array}$ & 2268 (19.29\%) & 687 (27.36\%) & & $458(26.77 \%)$ & 461 (26.94\%) & \\
\hline $\begin{array}{l}\text { Alcohol } \\
\text { consumption }\end{array}$ & & & 4.6 & & & 10.7 \\
\hline Non & 9717 (82.63\%) & 2085 (83.03\%) & & 1372 (80.19\%) & 1385 (80.95\%) & \\
\hline \multicolumn{7}{|c|}{ Values were $\mathrm{n}(\%)$ or mean \pm SD or median (interquartile range: 25 th to 75 th percentiles) } \\
\hline $\begin{array}{l}\text { SD, standard de } \\
\text { pressure; FPG, f } \\
\text { aminotransfera } \\
\text { cholesterol. }\end{array}$ & $\begin{array}{l}\text { ation; BMI, body ma } \\
\text { sting plasma glucos } \\
\text {;GT, gamma-gluta }\end{array}$ & $\begin{array}{l}\text { s index; WC, wai } \\
\text {; HbA1c,glycosyl } \\
\text { nyl transferase; }\end{array}$ & $\begin{array}{l}\text { circumfere } \\
\text { ed haemog } \\
\text { total chol }\end{array}$ & $\begin{array}{l}\text {; SBP, systolic blood } \\
\text {; ALT, alanine aminot } \\
\text { erol; TG,triglyceride; }\end{array}$ & $\begin{array}{l}\text { nsure; DBP, diast } \\
\text { nsferase; AST, as } \\
-C \text {, high-density }\end{array}$ & $\begin{array}{l}\text { lic blood } \\
\text { partate } \\
\text { poprotein }\end{array}$ \\
\hline
\end{tabular}




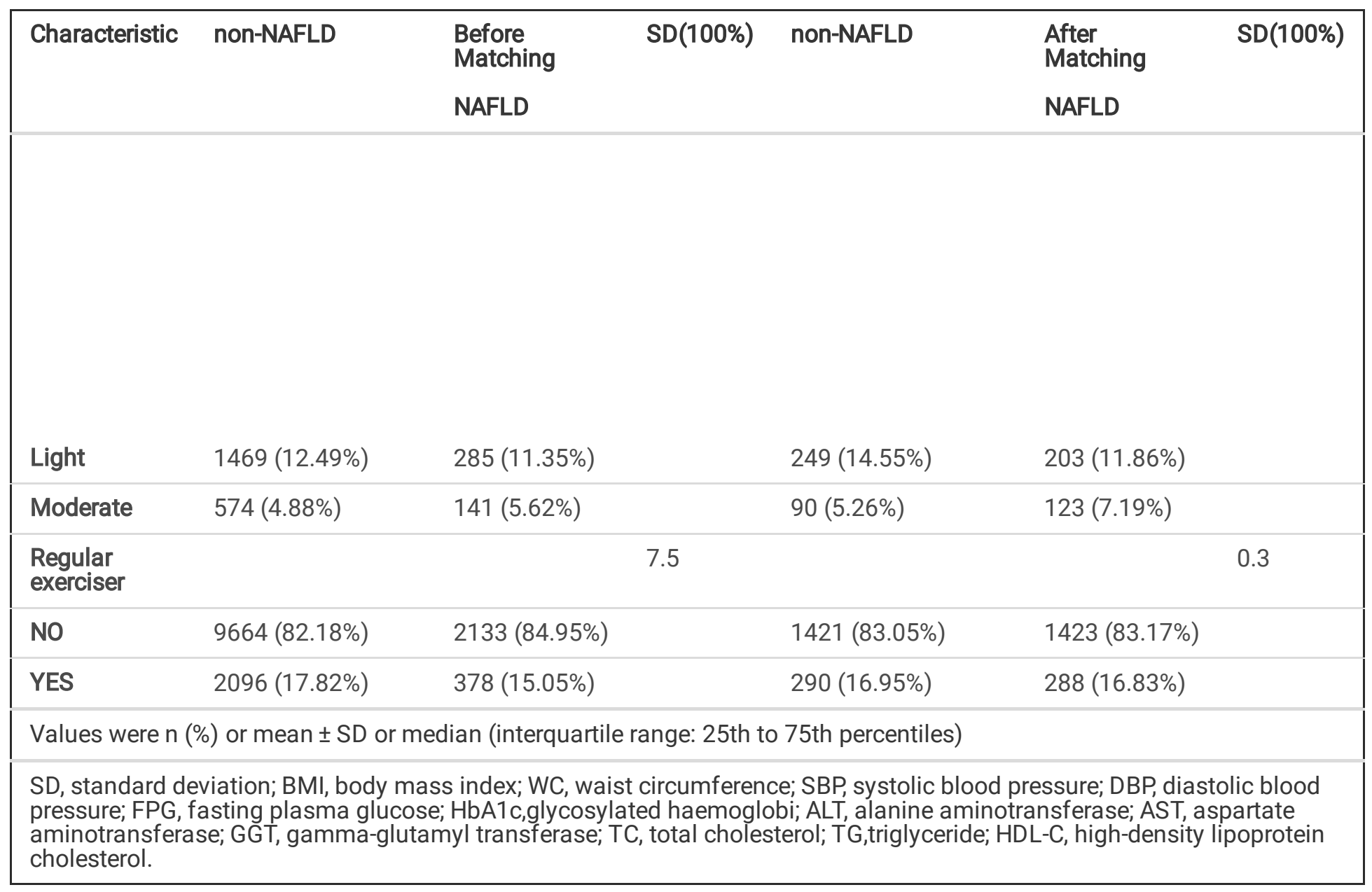

Besides, our study employed the Kaplan-Meier method to assess the incidence of diabetes in each subgroup and conducted the log-rank test to determine significance. The doubly robust estimation method, which combines propensity score models and outcome regression, verified the association between NAFLD and incidence of DM[21, 22]. To make our conclusions more reliable, we also used the Cox proportional hazards regression model to adjust variable imbalance between the NAFLD and non-NAFLD groups in the PS-matched cohort. According to the characteristics of diabetes risk, we conducted prespecified subgroup analyses. Division of subgroups was based on gender, WC, BMI, AST, ALT, TC, GGT, HbA1C, FPG, HDL-C, TG and propensity score. We converted continuous variables to categorical variables based on clinically significant cut-off point or median. In order to maintain the baseline balance between the NAFLD and non-NAFLD groups, we only selected the corresponding matched pairs in the subgroup. Likelihood ration tests inspected the modifications and interactions of subgroups.

For sensitivity analysis, this study used the PS obtained by logistic regression to estimate the inverse probability of treatment weights (IPTW). For the IPTW, NAFLD participants' weight was 1/PS, and non-NAFLD participants' weight was $1 /(1$ - PS). We used the IPTW model to create a weighted cohort[22]. Various sensitivity analysis methods were used to test the robustness of the research results. Two association inference models were added to the original cohort, and the weighted cohort was added to the sensitivity analysis. The effect sizes and $p$ values were calculated in all models. The results of our retrospective cohort study followed the STROBE statement[22].

The current research analysis was performed using the Empower-Stats (http://www.empowerstats.com, X\&Y Solutions, Inc., Boston, MA) and statistical software package R (http://www.R-project.org,The R Foundation). A two-sided $\mathrm{P}<0.05$ was considered significant.

\section{Results}




\section{Study population}

A total of 14,271 participants were eventually enrolled in our study, including $52.07 \%$ men and $47.93 \%$ women (Fig. 1). Among them, 2511 (17.60\%) participants had NAFLD and 11760 participants $(82.40 \%)$ did not suffer from NAFLD. The average age of the study population was $43.53 \pm 8.90$ years. During a median follow-up of $2206.64 \pm 1376.42$ days, 324 participants developed DM. Some baseline variables were statistically significant differences between NAFLD participants and non-NAFLD participants before conducting PSM. Higher age, BMI, WC, SBP, DBP, FPG, HbA1c, AST, ALT, GGT, TC and TG were observed in participants with NAFLD. We also found participants with NAFLD had a higher proportion of males, ever smoker and current smoker. Participants with non-NAFLD had a higher level of HDL-C and rates of regular exerciser. 1711 NAFLD patients were matched with 1711 non-NAFLD subjects by using one-to-one PSM. The standardized differences of almost all variables were less than $10.0 \%$ after PSM, showing an exact match. In other words, the differences in baseline characteristics between the two groups were minimal.

\section{The Incidence Of Diabetes}

The incidence of DM caused by NAFLD exposure before and after PSM was shown in Table 2. Our study included a total of 14,271 participants, 324 of whom developed DM during follow-up before PSM. The diabetes incidence rate in the overall population, NAFLD participants and non-NAFLD participants were 375.536/ 100,000 person-years, 1354.974/100,000 personyears and 168.512/100,000 person-years. NAFLD group and non-NAFLD group corresponding cumulative incidence of DM were 8.124 (7.055-9.194) and 1.020 (0.839-1.202), respectively. After PSM, the approximate incidence difference between the two groups changed greatly $(687.960 / 100,000$ person-years in the overall population, $907.082 / 100,000$ person-years in the NAFLD participants, and $458.980 / 100,000$ person-years in the non-NAFLD participants). The corresponding cumulative incidence in the NAFLD and non-NAFLD groups were 5.552 (4.466-6.638) and 2.688 (1.921-3.456).

Table 2

Incidence of incident DM before and after propensity score matching.

\begin{tabular}{|lllll|}
\hline Variable & Participants(n) & DM events(n) & $\begin{array}{l}\text { Cumulative incidence } \\
(95 \% \text { Cl) }\end{array}$ & Per 100,000 person-year \\
\hline Before Matching & & & & \\
\hline Total & 14271 & 324 & $2.270(2.026-2.515)$ & 375.536 \\
\hline NAFLD & 2511 & 204 & $8.124(7.055-9.194)$ & 1354.974 \\
\hline Non- NAFLD & 11760 & 120 & $1.020(0.839-1.202)$ & 168.512 \\
\hline After Matching & & & & \\
\hline Total & 3422 & 141 & $4.120(3.454-4.787)$ & 687.960 \\
\hline NAFLD & 1711 & 95 & $5.552(4.466-6.638)$ & 907.082 \\
\hline Non- NAFLD & 1711 & 46 & $2.688(1.921-3.456)$ & 458.980 \\
\hline Cl, confidence interval; DM, diabetes mellitus. & & \\
\hline
\end{tabular}

Kaplan-Meier analysis revealed that the cumulative incidence of DM in the participants with NAFLD was significantly higher than that in non-NAFLD participants before PSM $(p<0.0001$ by the log-rank; Fig. $2 a)$. There are still significant differences in the cumulative incidence of diabetes between the two groups in the PSM cohort (log-rank test; $P<0.0001 ;$ Fig. 2b). Besides, we also found that the cumulative incidence of diabetes was significantly higher in participants with higher propensity scores(Fig. 3).

\section{Association Between Nafld And Incident Diabetes}


The Cox proportional hazards regression model was applied to assess the association between NAFLD and DM risk in the PSM cohort. Table 3 showed the unadjusted, partially adjusted, fully adjusted and propensity-score adjusted models in detail. NAFLD was significantly associated with the incidence of DM in the unadjusted model $(\mathrm{HR}=1.92,95 \% \mathrm{Cl}$ : $1.35-2.74, \mathrm{P}=$ 0.0003). In other words, participants with NAFLD were 1.92 times more likely to develop diabetes than non-NAFLD participants. The results remained significant after adjusting for the partial confounding variables (age, gender, BMI, waist circumference, smoking status, alcohol consumption, regular exerciser, SBP, DBP) (HR: 2.00, 95\% Cl: $1.40-2.84, \mathrm{P}=0.0001$ ). In the fully adjusted model (adjusted for age, gender, BMI, WC, smoking status, alcohol consumption, regular exerciser, SBP, DBP, ALT, AST, GGT, HbA1c, FPG, TC, TG, HDL-C), we could still observe the association between NAFLD and incidence of DM (HR $=2.15$, 95\% Cl: $1.48-3.11, \mathrm{P}<0.0001)$. It showed that participants with NAFLD were 2.15 -fold more likely to develop diabetes than nonNAFLD participants. This association was still detected in the PSM model, and participants with NAFLD had an $89 \%$ increased risk of diabetes $(\mathrm{HR}=1.89,95 \% \mathrm{Cl}: 1.33-2.69, \mathrm{P}=0.0004)$.

Table 3

Association between NAFLD and incident diabetes in different models.

\begin{tabular}{|c|c|c|c|c|}
\hline Variable & $\begin{array}{l}\text { Non- } \\
\text { adjusted(HR,95\%Cl,P) }\end{array}$ & Model I (HR,95\%Cl, P) & Model II (HR,95\%Cl, P) & Model III (HR,95\%Cl, P) \\
\hline $\begin{array}{l}\text { Non- } \\
\text { NAFLD }\end{array}$ & Ref. & Ref. & Ref. & Ref. \\
\hline NAFLD & $1.92(1.35,2.74) 0.0003$ & $\begin{array}{l}2.00(1.40,2.84) \\
0.0001\end{array}$ & $\begin{array}{l}2.15(1.48,3.11)< \\
0.0001\end{array}$ & $\begin{array}{l}1.89(1.33,2.69) \\
0.0004\end{array}$ \\
\hline
\end{tabular}

Crude model: we did not adjust for other covariates.

Model I: we adjusted for age, gender, BMI, waist circumference, smoking status, alcohol consumption, regular exerciser, SBP, DBP.

Model Il: we adjusted for age, gender, BMI, waist circumference, smoking status, alcohol consumption, regular exerciser, SBP, DBP, ALT, AST, GGT, HbA1C, FPG, TC, TG, HDL-C.

Model III: we adjusted for propensity score.

HR, Hazard ratios; $\mathrm{Cl}$, Confidence interval; Ref, Reference

\section{Subgroup Analysis}

Subgroup analysis was applied to discover potential confounding variables that might affect the association between NAFLD and DM risk. To assess the trend of effect size in potential confounding variables, we used gender, BMI, WC, TC, TG, HDL-C, FPG, HbA1c, ALT, AST, GGT and propensity score as stratification variables. Table 4 revealed that most potential confounding variables did not influence the association between NAFLD and DM risk after PSM, except for BMI (P for interaction $=0.0140$ ) and visceral fat obesity ( $P$ for interaction $=0.0157)$. Specifically, compared with non-NAFLD participants with a $B M l<25 \mathrm{~kg} / \mathrm{m} 2$, the hazard ratios of $\mathrm{BMI}<25 \mathrm{~kg} / \mathrm{m} 2$ and $\mathrm{BMI} \geq 25 \mathrm{~kg} / \mathrm{m} 2$ in the NAFLD participants were $1.22(0.64,2.30)$, and 4.77 (1.98, 11.49), respectively. Concerning the non-NAFLD participants without the visceral fat obesity, the hazard ratios of non-visceral fat obesity and visceral fat obesity in the NAFLD participants were1.25 $(0.74,2.11)$ and $10.95(1.57,76.47)$. Thus, there was a more significant association between NAFLD and incidence of DM in the participants with $B M I \geq 25 \mathrm{~kg} / \mathrm{m}^{2}$ or visceral fat obesity. 
Table 4

Effect size of NAFLD on incident diabetes in prespecified and exploratory subgroups

\begin{tabular}{|c|c|c|c|c|}
\hline Characteristic & No of participants & $\mathrm{HR}(95 \% \mathrm{Cl})$ & $P$ value & $P$ for interaction \\
\hline Gender & & & & 0.6889 \\
\hline Male & 2082 & $1.86(1.15,2.98)$ & 0.0107 & \\
\hline Female & 218 & $164.87(0.00$, Inf $)$ & 0.9997 & \\
\hline $\mathrm{BMI}\left(\mathrm{Kg} / \mathrm{m}^{2}\right)$ & & & & 0.0140 \\
\hline$<25$ & 1580 & $1.22(0.64,2.30)$ & 0.5461 & \\
\hline$\geq 25$ & 668 & $4.77(1.98,11.49)$ & 0.0005 & \\
\hline Visceral fat obesity & & & & 0.0157 \\
\hline NO & 2008 & $1.25(0.74,2.11)$ & 0.4145 & \\
\hline YES & 242 & $10.95(1.57,76.47)$ & 0.0158 & \\
\hline FPG (mg/dL) & & & 0.8589 & \\
\hline Low & 786 & $2.74(0.69,10.93)$ & 0.1533 & \\
\hline High & 1056 & $2.43(1.43,4.16)$ & 0.0011 & \\
\hline HbA1c (\%) & & & & 0.8433 \\
\hline Low & 522 & $4.19(0.16,111.82)$ & 0.3929 & \\
\hline High & 1306 & $2.48(1.55,3.98)$ & 0.0002 & \\
\hline TC (mg/dL) & & & & 0.5314 \\
\hline Low & 792 & $1.83(0.55,6.05)$ & 0.3248 & \\
\hline High & 834 & $2.94(1.54,5.61)$ & 0.0011 & \\
\hline TG (mg/dL) & & & & 0.3118 \\
\hline Low & 970 & $1.45(0.62,3.38)$ & 0.3914 & \\
\hline High & 990 & $2.65(1.44,4.87)$ & 0.0018 & \\
\hline $\mathrm{HDL}-\mathrm{C}(\mathrm{mg} / \mathrm{dL})$ & & & & 0.4346 \\
\hline Low & 944 & $3.42(1.76,6.66)$ & 0.0003 & \\
\hline High & 950 & $6.36(1.61,25.06)$ & 0.0082 & \\
\hline $\operatorname{ALT}(\mathrm{U} / \mathrm{L})$ & & & & 0.5752 \\
\hline Low & 974 & $2.29(0.85,6.15)$ & 0.0995 & \\
\hline High & 1006 & $3.08(1.59,5.99)$ & 0.0009 & \\
\hline $\mathrm{AST}(\mathrm{U} / \mathrm{L})$ & & & & 0.8560 \\
\hline Low & 822 & $2.43(0.81,7.29)$ & 0.1134 & \\
\hline High & 1030 & $2.60(1.34,5.03)$ & 0.0047 & \\
\hline GGT(U/L) & & & & 0.0986 \\
\hline
\end{tabular}

Note 1: The above model has been adjusted for age, gender, BMI, waist circumference, smoking status, alcohol consumption, regular exerciser, SBP, DBP, ALT, AST, GGT, HbA1c, FPG, TC, TG, HDL-C. 


\begin{tabular}{|c|c|c|c|c|}
\hline Characteristic & No of participants & $\mathrm{HR}(95 \% \mathrm{Cl})$ & $P$ value & $P$ for interaction \\
\hline Low & 934 & $6.55(1.81,23.71)$ & 0.0042 & \\
\hline High & 988 & $2.16(1.19,3.92)$ & 0.0111 & \\
\hline propensity score & & & & 0.4530 \\
\hline Low & 1684 & $1.61(0.76,3.40)$ & 0.2121 & \\
\hline High & 1684 & $2.28(1.47,3.53)$ & 0.0002 & \\
\hline
\end{tabular}

\section{Sensitivity Analysis}

We applied the estimated propensity score as the weight and generated a weighted cohort by establishing an IPTW model. Our study evaluated the association between NAFLD and incidence of DM in the original cohort and the weighted cohort through the Cox proportional hazards regression model, which could increase the robustness of results. Besides, the unadjusted, partially and fully adjusted models were established in both cohorts in Table 5. We demonstrated that NAFLD was closely associated with the risk of DM in the original cohort or the weighted cohort. In the fully adjusted models, participants with NAFLD had an $82 \%$ increase in the risk of DM in the original cohort ( $\mathrm{HR}=1.82,95 \% \mathrm{Cl}: 1.33-2.48, \mathrm{P}=0.0001)$, and a $70 \%$ increase in the weighted cohort $(\mathrm{HR}=1.70,95 \% \mathrm{Cl}: 1.40-2.06, \mathrm{P}<0.00001)$.

Table 5 Association between NAFLD and incident diabetes in different models of the original and the weighted cohort.

A

\begin{tabular}{|llll|}
\hline Variable & Non-adjusted & Model I $(\mathrm{HR}, 95 \% \mathrm{Cl}, \mathrm{P})$ & Model II $(\mathrm{HR}, 95 \% \mathrm{Cl}, \mathrm{P})$ \\
\hline Non-NAFLD & Ref. & Ref. & Ref. \\
\hline NAFLD & $8.08(6.45,10.13)<0.0001$ & $3.84(2.92,5.05)<0.0001$ & $1.82(1.33,2.48) 0.0001$ \\
\hline
\end{tabular}

B

\begin{tabular}{|llll|}
\hline Variable & Non-adjusted & Model I $(\mathrm{HR}, 95 \% \mathrm{Cl}, \mathrm{P})$ & Model II $(\mathrm{HR}, 95 \% \mathrm{Cl}, \mathrm{P})$ \\
\hline Non-NAFLD & Ref. & Ref. & Ref. \\
\hline NAFLD & $3.06(2.60,3.60)<0.0001$ & $2.79(2.37,3.29)<0.0001$ & $1.70(1.40,2.06)<0.0001$ \\
\hline
\end{tabular}

A In the original cohort; B In the weighted cohort.

Crude model: we did not adjust for other covariates.

Model I: we adjusted for age, gender, BMI, waist circumference, smoking status, alcohol consumption, regular exerciser, SBP, DBP.

Model II: we adjusted for age, gender, BMI, waist circumference, smoking status, alcohol consumption, regular exerciser, SBP, DBP, ALT, AST, GGT, HbA1c, FPG, TC, TG, HDL-C.

HR, Hazard ratios; Cl, Confidence interval; Ref, Reference 


\section{Discussion}

The PSM cohort study indicated that NAFLD was an independent risk factor for developing DM after adjusting for confounding variables in our study. The risk of developing DM in the NAFLD participants increased by $92 \%$ in the PSM cohort. In the sensitivity analysis, participants with NAFLD had an $82 \%$ increase in the risk of DM in the original cohort and a $70 \%$ increase in the weighted cohort. It could be better understood the association between NAFLD and incidence of DM in different subjects by subgroups analysis. There was a stronger association between NAFLD and the incidence of DM in subjects with BMI $\geq$ $25 \mathrm{~kg} / \mathrm{m} 2$ or visceral fat obesity. In contrast, there was a weaker connection between NAFLD and incident of DM in the participants with $\mathrm{BMI}<25 \mathrm{~kg} / \mathrm{m} 2$ or non-visceral fat obesity.

NAFLD can progress to liver fibrosis, cirrhosis, liver cancer, and increase the risk of developing diabetes and cardiovascular disease[23]. Besides, NAFLD and diabetes have common risk factors and often occurred simultaneously or sequentially in one person[6]. However, more and more epidemiological evidence shows that NAFLD may be earlier than the development of $\mathrm{DM}[24,25]$. In a meta-analysis of 19 retrospective cohort studies involving nearly 300,000 individuals and approximately 16,000 cases of DM, participants with NAFLD had a higher incidence of DM compared with participants without NAFLD (HR $2.22 ; 95 \% \mathrm{Cl} 1.84-2.60$ )[26]. In a recent meta-analysis of 33 studies involving more than 500,000 individuals and approximately 28,000 diabetic participants, participants with NAFLD increased the risk of diabetes by 1.19 times compared with those without NAFLD[10]. In addition, a series of prospective studies showed that NAFLD strongly increased the incidence of $\mathrm{DM}[4,27,28]$. In contrast, some research demonstrated different findings that the connection between NAFLD and the risk of developing DM was not significant after adjusting for some confounding factor[29,30]. We speculated that the reasons for the inconsistent findings might have the following elements: (1) The study subjects were diverse, including race, gender, ethnicity, and age. (2)The sample size varies greatly between different studies. (3) Previous studies were adjusted for different confounding variables, which affected the connection between NAFLD and DM risk. (4) The follow-up time of these studies varied widely, which affected the incidence of DM. Our results enriched existing studies that supported the following conclusions: NAFLD increases the risk of developing DM.

In our study based on large-scale cohort data using propensity scores, we revealed that participants with NAFLD were 0.92 times more likely to develop diabetes than non-NAFLD. In this study, the risk of diabetes caused by NAFLD was lower than in previous researches. The possible reason for the inconsistency between our study and previous research might be that we conducted a PSM analysis to effectively control the impact of confounding variables. Therefore, our research better revealed the connection between NAFLD and DM. In addition, our study adjusted more confounding variables that affected NAFLD and diabetes. We adjusted for more the demographic and clinical variables, including age, gender, BMI, waist circumference, smoking status, alcohol consumption, regular exerciser, SBP, DBP, ALT, AST, GGT, HbA1c, FPG, TC, TG, HDL-C. There was evidence that these variables were related to NAFLD and DM[9, 28]. Besides, our study was based on large cohort data (14271 participants). The conclusions supported that NAFLD increased the risk of developing DM. Exploring more about the association between NAFLD and diabetes can help us better communicate risk to patients and make management strategies to reduce DM risk. In the past, PSM was mainly applied to compare different treatments[31, 32]. Our study contributed to the promotion of PSM methods in related research.

The mechanism of NAFLD leading to diabetes remained unclear, but NAFLD can cause IR, which is the essential pathogenesis of diabetes[33]. The following mechanism in the NAFLD may mediate the production of IR: (1) Adipose tissue dysfunction and inflammation promoted the secretion of adipokines, increased the secretion of pro-inflammatory cells (such as tumor necrosis factor-a), and increased the release of free fatty acid, resulting in decreased insulin sensitivity; Adipose tissue dysfunction and inflammation interfered with the activation of the pro-inflammatory pathway of insulin signal transmission, which directly led to a decrease in insulin sensitivity[34]. (2) Certain incretin related to NAFLD can directly inhibit the production of endogenous glucose through an insulin-dependent mechanism[35]. The reduction of these incretin effects also led to IR[35]. (3) Increased expression of dipeptidyl peptidase-4 could impair insulin sensitivity by reducing incretin levels and promoting liver disease progression through independent mechanisms[36, 37]. 


\section{Study Strengths And Limitations}

Our study had some strengths as follows. The innovation of this study is that the propensity score was used to explore the relationship between NAFLD and the risk of developing DM. In recent years, the propensity scoring method has been widely used in observational research. Its acknowledged advantages include a wide range of data requirements, reducing inter-group differences, balancing inter-group confounders and achieving the effect of "similar randomization". We also performed a subgroup analysis to reveal other potential risks of NAFLD and DM association in different subgroups. To ensure the robustness of the results, we performed a series of sensitivity analyses. We mainly used the IPTW to establish a weighted cohort and further explore the association between NAFLD and incidence of DM in the weighted cohort. More importantly, the size of the participants in our study was more extensive than most previous retrospective cohort studies.

On the contrary, the current study also had several limitations. First, the population included in our study was Japanese, so whether the conclusion could be generalized to all people needed further research. Second, the PS could only balance the known confounding factors and not consider the influence of unknown factors, such as the family history of diabetes. Third, the lack of a 2-hour oral glucose tolerance test in the original study might underestimate the incidence of DM. However, due to a lack of financial support, it was not feasible to conduct a 2-hour oral glucose tolerance test for all participants. Fourth, the PS could balance known confounding variables as much as possible, but it could not ensure that all measured baseline characteristics were matched and consider the influence of unknown variables. To reduce the interference of variables on the measurement results, we control the caliper width to 0.01. Fifth, PSM could lead to loss of information, which might change the composition of the sample. Sixth, the diagnosis of fatty liver was based on ultrasound examination, which was not as accurate as liver biopsy. Therefore, clinical trials were still needed to confirm NAFLD treatment's effectiveness in reducing the incidence of diabetes.

\section{Conclusion}

In the PSM cohort, the risk of developing DM in the participants with NAFLD increased by $92 \%$. In the sensitivity analysis, the risk of developing DM in the participants with NAFLD increased by $70 \%$ in the weighted cohort. Additionally, subgroup analysis showed that obese participants with NAFLD should be more concerned about the risk of diabetes.

\section{Abbreviations}

BMI, body mass index; WC, waist circumference; SBP, systolic blood pressure; DBP, diastolic blood pressure; FPG, fasting plasma glucose; HbA1c, glycosylated hemoglobin; ALT, alanine aminotransferase; AST, aspartate aminotransferase; GGT, gamma-glutamyl transferase; TC, total cholesterol; TG, triglyceride; HDL-C, high-density lipoprotein cholesterol; T2DM, type 2 diabetes mellitus; DM, diabetes mellitus; SD, standardized difference; HR, hazard ratios; Cl, Confidence intervals; Ref, reference; PS, propensity score; IPTW, inverse probability of treatment weights; NAFLD nonalcoholic fatty liver disease; IR, insulin resistance; NAGALA, NAfld in the Gifu Area, Longitudinal Analysis.

\section{Declarations}

\section{Acknowledgments}

Not applicable

\section{Authors' contributions}

Xiaodan Zheng and Changchun Cao contributed to the study's concept and design, researched and interpreted the data, and drafted the manuscript. Yongcheng He and Xinyu Wang analyzed data and reviewed the manuscript. Jun Wu and Haofei Hu were the guarantors of this work, had full access to all the data in the study, and took responsibility for the integrity of the data and accuracy of the data analysis. All authors read and approved the final manuscript.

\section{Funding}

Page $12 / 17$ 
This study was supported by the Discipline Construction Ability Enhancement Project of Shenzhen Municipal Health Commission (SZXJ2017031). This study was also supported by the International Cooperative Research Project of Shenzhen Municipal Science and Technology Innovation Council (accounts GJHZ2018041616481462).

\section{Availability of data and materials}

The data are available from the 'DataDryad' database (www.datadryad.org).

\section{Ethics approval and consent to participate}

The ethics committee approved the original research of Murakami Memorial Hospital, and informed consents were obtained from all participants

\section{Consent for publication}

Not applicable.

\section{Competing interests}

The authors declare that they have no competing interests.

\section{Author details}

${ }^{1}$ Department of Neurology, Peking University Shenzhen Hospital, Shenzhen 518000, Guangdong Province, China

${ }^{2}$ Department of Clinical Medicine, Shantou University Medical College, Shantou 515000, Guangdong Province, China

${ }^{3}$ Department of Rehabilitation, Shenzhen Dapeng New District Nan'ao People's Hospital, Shenzhen 518000, Guangdong Province, China

${ }^{4}$ Department of Nephrology, Shenzhen Hengsheng Hospital, Shenzhen 518000, Guangdong Province, China

${ }^{5}$ Department of Endocrinology, The First Affiliated Hospital of Shenzhen University, Shenzhen 518000, Guangdong Province, China

${ }^{6}$ Department of Nephrology, The First Affiliated Hospital of Shenzhen University, Shenzhen 518000, Guangdong Province, China

\section{References}

1. Saeedi P, et al. Global and regional diabetes prevalence estimates for 2019 and projections for 2030 and 2045: Results from the International Diabetes Federation Diabetes Atlas, 9(th) edition. Diabetes Res Clin Pract. 2019;157:107843.

2. Economic Costs of Diabetes in the U.S. in 2017. Diabetes Care, 2018. 41(5): p. 917-928.

3. Ali MK, et al., Diabetes: An Update on the Pandemic and Potential Solutions. 2017.

4. Chen GY, et al. New risk-scoring system including non-alcoholic fatty liver disease for predicting incident type 2 diabetes in East China: Shanghai Baosteel Cohort. J Diabetes Investig. 2016;7(2):206-11.

5. Ma J, et al. Bi-directional analysis between fatty liver and cardiovascular disease risk factors. J Hepatol. 2017;66(2):3907.

6. Younossi ZM, et al. Global epidemiology of nonalcoholic fatty liver disease-Meta-analytic assessment of prevalence, incidence, and outcomes. Hepatology. 2016;64(1):73-84.

7. Stefan N, Haring HU, Cusi K. Non-alcoholic fatty liver disease: causes, diagnosis, cardiometabolic consequences, and treatment strategies. Lancet Diabetes Endocrinol. 2019;7(4):313-24. 
8. Liu M, et al. Association of NAFLD With Diabetes and the Impact of BMI Changes: A 5-Year Cohort Study Based on 18,507 Elderly. J Clin Endocrinol Metab. 2017;102(4):1309-16.

9. Chen SC, et al, Fat L, Enzymes H. Alkaline Phosphatase and the Risk of Incident Type 2 Diabetes: A Prospective Study of 132,377 Adults. Sci Rep. 2017;7(1):4649.

10. Mantovani A, et al., Non-alcoholic fatty liver disease and risk of incident diabetes mellitus: an updated meta-analysis of 501022 adult individuals. Gut, 2020.

11. Siu JM, et al. Association of Vessel-Sealant Devices vs Conventional Hemostasis With Postoperative Neck Hematoma After Thyroid Operations. JAMA Surg. 2019;154(11):e193146.

12. Okamura T, et al. Ectopic fat obesity presents the greatest risk for incident type 2 diabetes: a population-based longitudinal study. Int J Obes (Lond). 2019;43(1):139-48.

13. Chitturi S, et al. Non-alcoholic fatty liver disease in the Asia-Pacific region: definitions and overview of proposed guidelines. J Gastroenterol Hepatol. 2007;22(6):778-87.

14. Hashimoto $Y$, et al. Modest alcohol consumption reduces the incidence of fatty liver in men: a population-based largescale cohort study. J Gastroenterol Hepatol. 2015;30(3):546-52.

15. Ryu S, et al. gamma-Glutamyltransferase as a predictor of chronic kidney disease in nonhypertensive and nondiabetic Korean men. Clin Chem. 2007;53(1):71-7.

16. Misra A, et al. Waist circumference cutoff points and action levels for Asian Indians for identification of abdominal obesity. Int J Obes (Lond). 2006;30(1):106-11.

17. Basevi V, et al., Comment on: American Diabetes Association. Standards of medical care in diabetes-2011. Diabetes Care 2011;34(Suppl. 1):S11-S61. Diabetes Care, 2011. 34(5): p. e53; author reply e54.

18. Normand ST, et al. Validating recommendations for coronary angiography following acute myocardial infarction in the elderly: a matched analysis using propensity scores. J Clin Epidemiol. 2001;54(4):387-98.

19. Ahmed A, et al. Heart failure, chronic diuretic use, and increase in mortality and hospitalization: an observational study using propensity score methods. Eur Heart J. 2006;27(12):1431-9.

20. Guo C, et al. Association between mean arterial pressure and risk of type 2 diabetes mellitus: The Rural Chinese Cohort Study. Prim Care Diabetes. 2020;14(5):448-54.

21. McCaffrey DF, et al. A tutorial on propensity score estimation for multiple treatments using generalized boosted models. Stat Med. 2013;32(19):3388-414.

22. Koch B, Vock DM, Wolfson J. Covariate selection with group lasso and doubly robust estimation of causal effects. Biometrics. 2018;74(1):8-17.

23. Chalasani $\mathrm{N}$, et al. The diagnosis and management of nonalcoholic fatty liver disease: Practice guidance from the American Association for the Study of Liver Diseases. Hepatology. 2018;67(1):328-57.

24. Lonardo A, et al. Hypertension, diabetes, atherosclerosis and NASH: Cause or consequence? J Hepatol. 2018;68(2):33552.

25. Byrne CD, Targher G. NAFLD: a multisystem disease. J Hepatol. 2015;62(1 Suppl):S47-64.

26. Mantovani A, et al. Nonalcoholic Fatty Liver Disease and Risk of Incident Type 2 Diabetes: A Meta-analysis. Diabetes Care. 2018;41(2):372-82.

27. Liu M, et al. Association of NAFLD With Diabetes and the Impact of BMI Changes: A 5-Year Cohort Study Based on 18,507 Elderly. J Clin Endocrinol Metab. 2017;102(4):1309-16.

28. Li Y, et al. Bidirectional association between nonalcoholic fatty liver disease and type 2 diabetes in Chinese population: Evidence from the Dongfeng-Tongji cohort study. PLoS One. 2017;12(3):e0174291.

29. Adams LA, et al. NAFLD as a risk factor for the development of diabetes and the metabolic syndrome: an eleven-year follow-up study. Am J Gastroenterol. 2009;104(4):861-7.

30. Okamoto M, et al. The association of fatty liver and diabetes risk. J Epidemiol. 2003;13(1):15-21. 
31. Bae J, et al. Differential Effects of Thiazolidinediones and Dipeptidyl Peptidase-4 Inhibitors on Insulin Resistance and beta-Cell Function in Type 2 Diabetes Mellitus: A Propensity Score-Matched Analysis. Diabetes Ther. 2019;10(1):149-58.

32. Wu T, et al. Five-year effectiveness of bariatric surgery on disease remission, weight loss, and changes of metabolic parameters in obese patients with type 2 diabetes: A population-based propensity score-matched cohort study. Diabetes Metab Res Rev. 2020;36(3):e3236.

33. Tilg H, Moschen AR, Roden M. NAFLD and diabetes mellitus. Nat Rev Gastroenterol Hepatol. 2017;14(1):32-42.

34. Najjar SM, Perdomo G. Hepatic Insulin Clearance: Mechanism and Physiology. Physiology (Bethesda), 2019. 34(3): p. 198-215.

35. Jun LS, et al. Absence of glucagon and insulin action reveals a role for the GLP-1 receptor in endogenous glucose production. Diabetes. 2015;64(3):819-27.

36. Miyazaki M, et al. Increased hepatic expression of dipeptidyl peptidase-4 in non-alcoholic fatty liver disease and its association with insulin resistance and glucose metabolism. Mol Med Rep. 2012;5(3):729-33.

37. Itou M, et al. Dipeptidyl peptidase-4: a key player in chronic liver disease. World J Gastroenterol. 2013;19(15):2298-306.

\section{Figures}

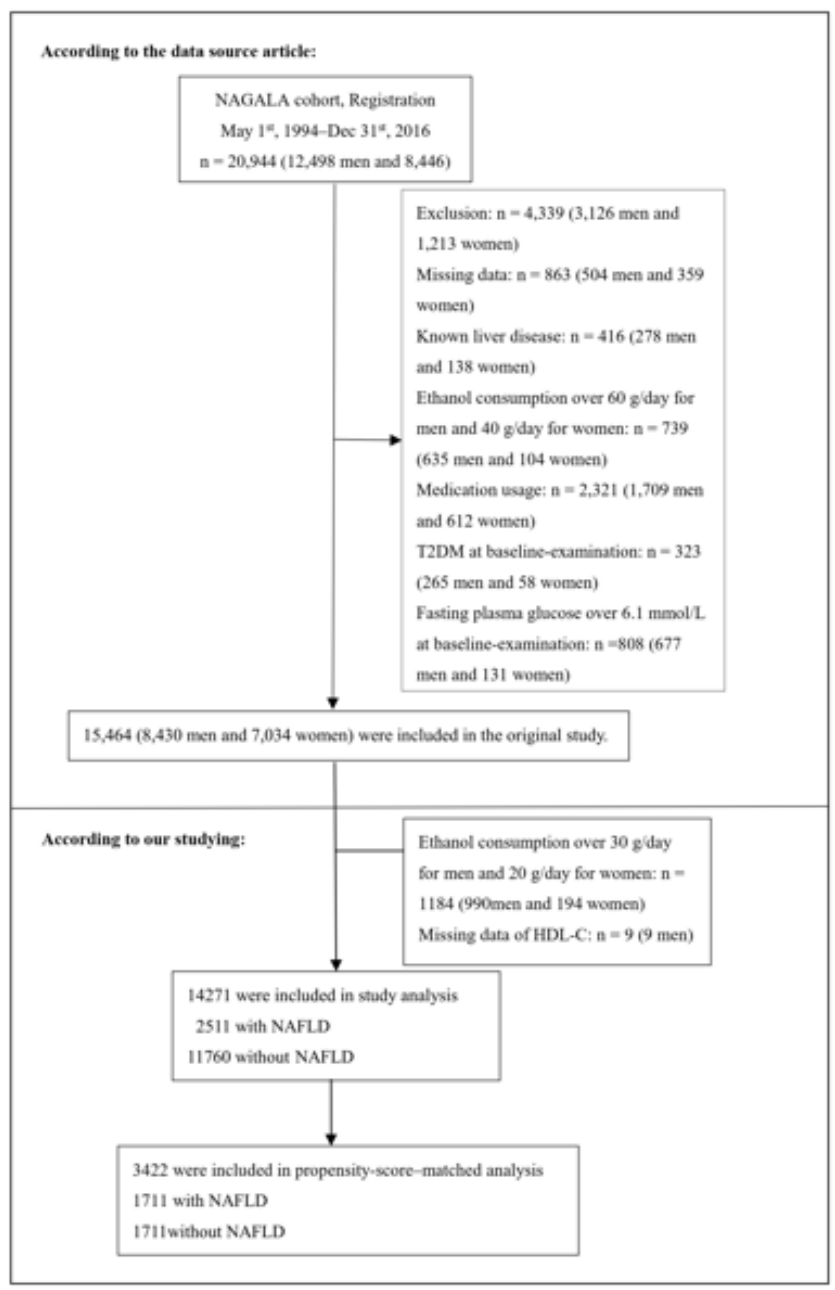

\section{Figure 1}

Study Population 

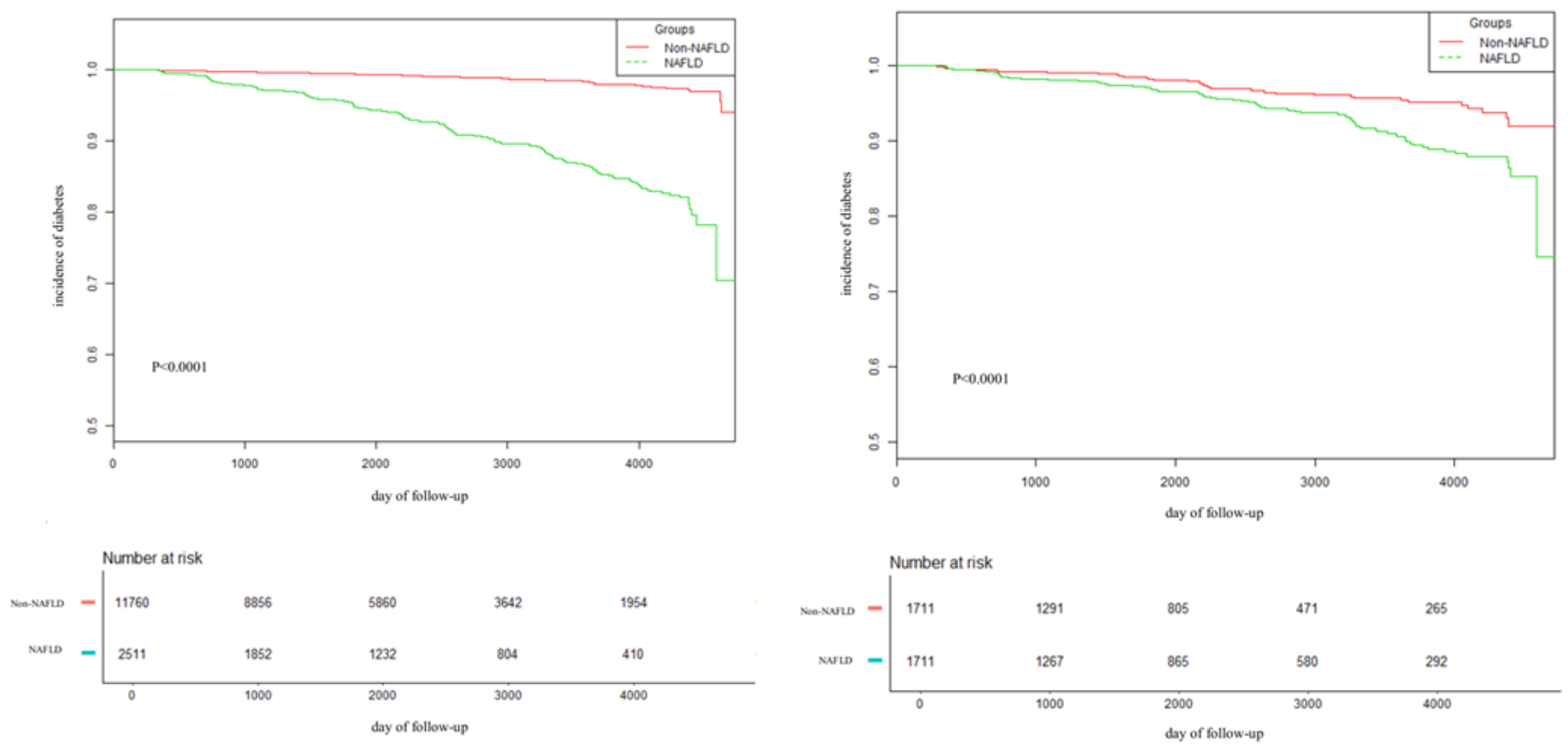

A

B

Figure 2

a Kaplan-Meier analysis of incident diabetes based on NAFLD and non- NAFLD in the original cohort (log-rank, P $<0.0001)$. b Kaplan-Meier analysis of incident diabetes based on NAFLD and non-NAFLD in the propensity score matching cohort (logrank, $\mathrm{P}<0.0001)$.
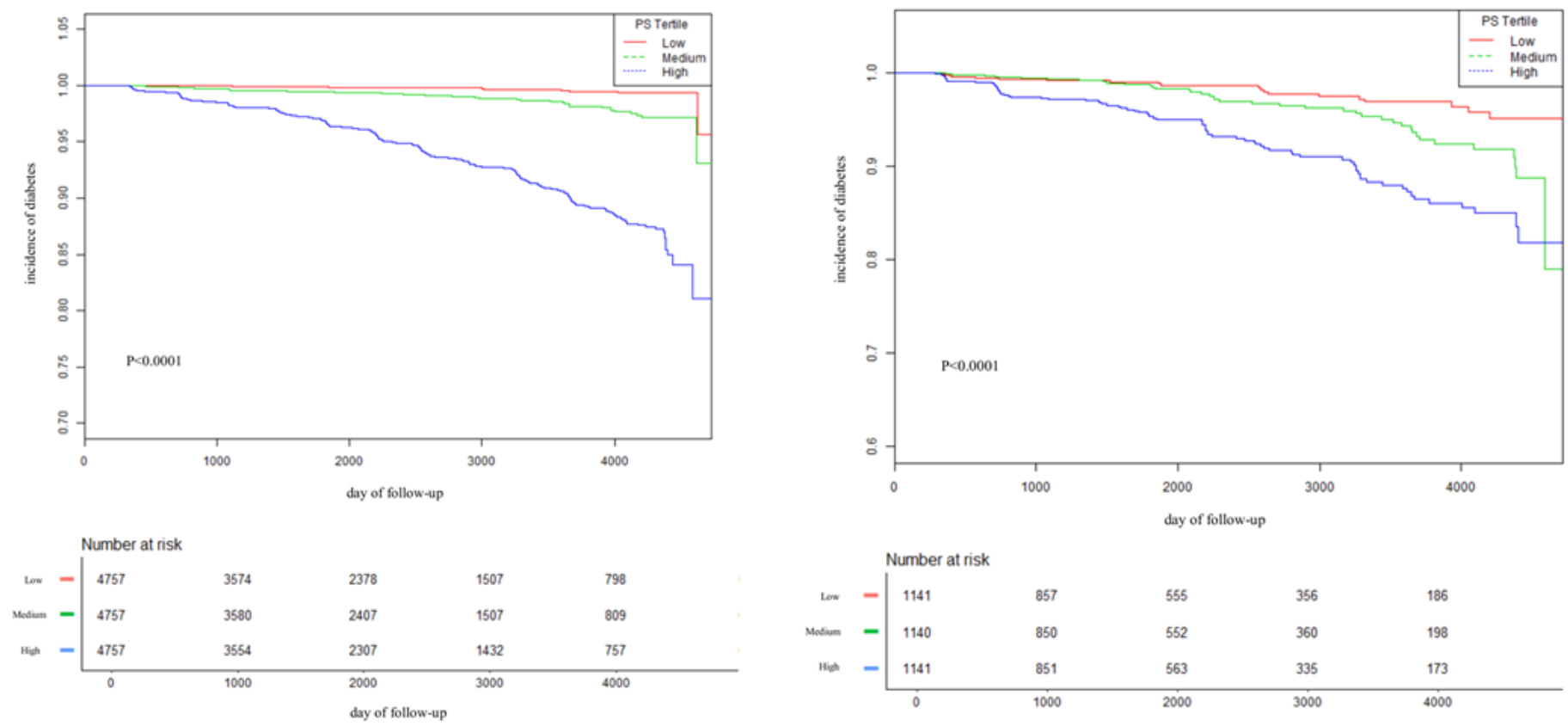

A

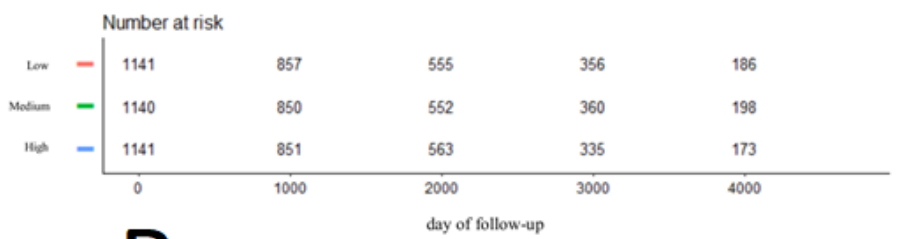

Figure 3 
a Kaplan-Meier analysis of incident diabetes based on propensity score (PS) tertile in the original cohort (log-rank, P < 0.0001). b Kaplan-Meier analysis of incident diabetes based on propensity score (PS) tertile in the propensity score matching cohort (log-rank, $\mathrm{P}<0.0001)$. 